\title{
DEVELOPMENT OF FUZZY BLOCKS INTENDED FOR AGENTS CONSTITUTING INTELLIGENT SYSTEM OF MOBILE ROBOT MOVEMENT PLANNING
}

\author{
Andrius NEMEIKŠIS, Faculty of Engineering, University of Life Sciences and Technologies, 5 J. Cakstes blvd., Jelgava LV-3001, Latvia; \\ nemeiksis.andrius@llu.lv (corresponding author) \\ Vitalijs OSADČUKS, Faculty of Engineering,University of Life Sciences and Technologies, 5 J. Cakstes blvd., Jelgava LV-3001, Latvia; \\ vitalijs.osadcuks@1lu.lv
}

\begin{abstract}
With the advancement of technologies, there are attempts to automate the majority of processes for various reasons, for instance, to improve and optimize production or to perform actions that may cause risk to people's health, etc. Therefore, the use of mobile autonomous robots is becoming increasingly important as the limits of the potential of the use of autonomous mobile robots in the industry have not yet been reached. The attempts have been made to achieve this by developing optimum trajectory calculation algorithms which enable the robot to move freely in both static and dynamic environments and use an optimum trajectory. Therefore, the subject of study in this article was movement of a mobile robot in an unknown environment using a multi-agent device system and fuzzy logics, and the goal of the study was to prepare the methods for development of intelligent systems for planning mobile robot movement in an unknown environment using multi-agent device and fuzzy logics ensuring the robot will accomplish the planned and adjusted on the go safe trajectory in the environment with unknown obstacles. Based on this, the robot arm model has been developed after calculating in the article the missing parameters of the experimental mobile robot in order to analyze the peculiarities of using the multi-agent device as well as the specifics and challenges of using fuzzy logics. As a result of the study performed in the article, significant data were obtained based on which a method was offered for an intelligent system for planning mobile robot movement in an unknown static environment using a multi-agent system, which was characterized by the use of fuzzy blocks corresponding each agent, and localization of each solution to the task of planning robot movement in each specific situation, which enables to improve the accuracy and efficiency of movement planning.
\end{abstract}

Keywords: unknown environment, static obstacles, multi-agent system, planned track, mobile robot, fuzzy logic.

\section{INTRODUCTION}

In modern robotics there is a topical issue related to the simplification of real-time calculations so that a robot could quickly perform response movements in the event of a collision with an obstacle. For this case the purpose of planning is to calculate a new robot path in a real-time mode without much delay. This issue is tackled by using fuzzy logic. The use of fuzzy logic is directly related to the use of traditional models for making decisions under the conditions of fast-moving non-linear processes, where quantitative methods are not acceptable (Luo, 2017; Adib, 2017). The use of fuzzy logic in this case is characterized by a relatively low cost compared to other methods, the ability to generate prompt response movements in realtime mode and, furthermore, it is used when the certain type of control object or process is not identified.

The use of fuzzy logic is described in various studies aimed at the analysis of issues related to the mobile robot motion planning in familiar and unfamiliar environments (Thinh, 2016; Ahmad, 2016). Therefore, the study object of this article is the movement of a mobile robot under the conditions of unfamiliar environment by using a multi-agent apparatus system (Berisha, 2016; Handayani, 2017) and fuzzy logic (Boujelben, 2017; Nasrinahar, 2016). The article analyses the movement of a mobile robot in an unfamiliar static environment using an intelligent multi-agent planning system and its fuzzy structure consisting of four fuzzy blocks for each of the indicated agents and a safe movement agent. The study analyses a mobile robot that performs three tasks: bypassing the obstacles, tracking the wall and moving towards the destination point. Therefore, the article aims to assess whether the application of a multi-agent method with fuzzy logic system in developing an intelligent planning system is optimal for dealing with the mobile robot motion planning task in an unfamiliar static environment, and to analyse the configuration path of such system for the accuracy and efficiency of motion planning and the logical accuracy of the intelligent planning system.

Copyright () 2019 The Authors. Published by Vytautas Magnus University. This is an open-access article distributed under the terms of the Creative Commons Attribution License (CC BY 4.0), which permits unrestricted use, distribution, and reproduction in any medium, provided the original author and source are credited. 


\section{MATERIALS AND METHODS}

Intelligent system of planning mobile robot movement in an unknown environment using multi-agent system is based on four agents: movement-to-destination agent, wall tracking agent, obstacle-bypass agent and speed control agent. The structural scheme of the intelligent system for planning mobile robot movement using multi-agent system presented in Fig. 1.

Intelligent system inputs $\left(\mathrm{x}_{\mathrm{t}}, \mathrm{y}_{\mathrm{t}}\right)$ are the coordinates of the destination point. Intelligent system outputs $\left(\mathrm{x}_{\mathrm{g}}, \mathrm{y}_{\mathrm{g}}\right)$ are the coordinates of the mobile robot current point. Based on the values of $\Delta \mathrm{x}_{\mathrm{t}}$ and $\Delta \mathrm{y}_{\mathrm{t}}$ axis deviations, the distance $d_{\text {destination }}$ and deviation angle $\theta$ between the robot and destination point are determined. Angles $\mu_{1}, \mu_{2}$ and $\mu_{3}$ are mobile robot rotation angles in respectively obstacle-bypass agent, wall tracking agent, and movement-to-destination agent. $\mathrm{V}_{\mathrm{L}}, \mathrm{V}_{\mathrm{R}}-$ mobile robot left and right wheel velocities.

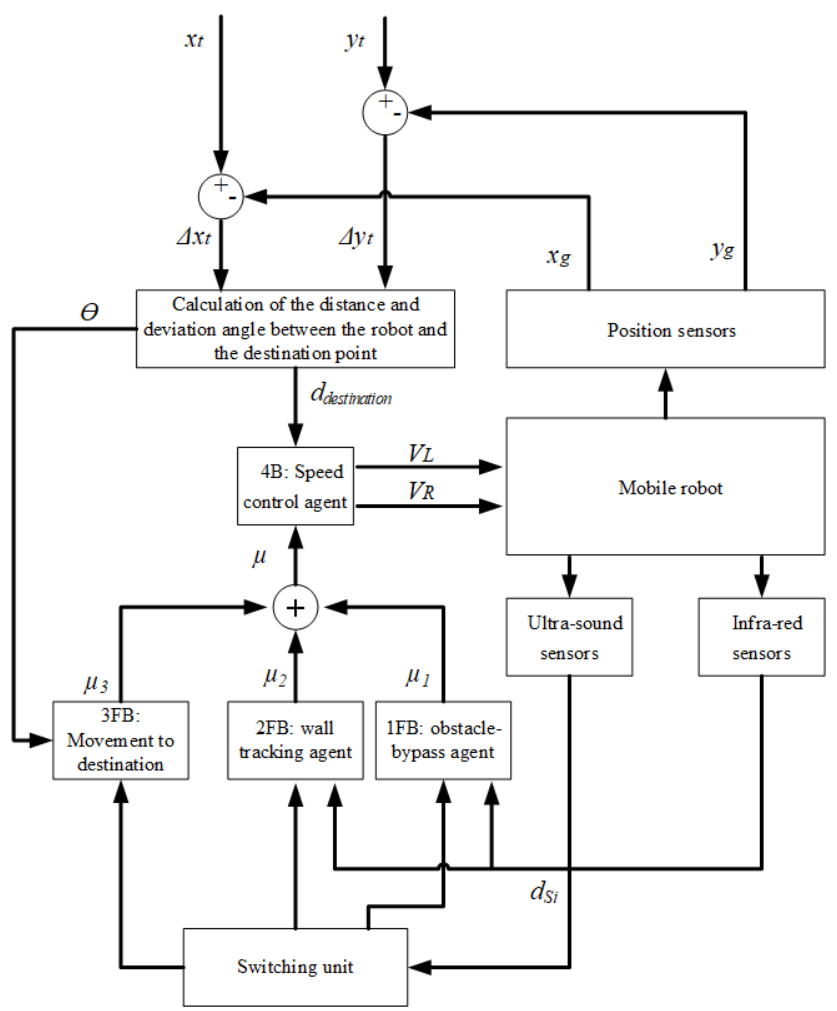

Figure 1. Structural scheme of an intelligent system for planning mobile robot movement in an unknown static environment using multiagent system

In this system, four fuzzy blocks (FB) are used to accomplish each agent's task: FB-1 for obstacle-bypass agent; FB-2 for wall tracking agent; FB-3 for movement-to-destination agent and FB-4 for speed control agent. The switch unit constantly detects the situation of the agents, activates and switches agents. Development of this system is based on a three-stage process.

During the first stage, the value of the distance between the robot and the obstacles in its operating area $\left(\mathrm{d}_{\mathrm{S1}}\right)$ is determined based on the information received from distance sensors; also the switch unit is developed.

The second stage involves development of obstacle-bypass agent, wall tracking agent, and movement-to-destination agent. Signals of the sensors US2-IR2, US3-IR3, US4-IR4 or US7-IR7, US8-IR8, US9-IR9 are the outputs of the first fuzzy block (1FB) which performs the role of obstacle-bypass agent. The second fuzzy block (2FB) - wall tracking agent - received signals from the sensors US1-IR1, US10-IR10 or US5-IR5, US6-IT6. Movement-to-destination agent is based on the third fuzzy block (3FB), when there are no obstacles around the robot in the sensor operation area. Fuzzy block outputs are the mobile robot rotation angles $\mu_{1}, \mu_{2}$ and $\mu_{3}$.

During the third stage, speed control agent is developed using the fourth fuzzy block (4FB). Based on the results obtained during the first and second stages, the final value of mobile robot rotation angle change $-\mu$, is determined corresponding to each of the agents. Mobile robot rotation angle and the distance between the robot and the destination point are the inputs of the fourth fuzzy block (4FB). Outputs of the 4FB: signal controlled by the drives which help to avoid collisions of the robot with unknown obstacles and reach the destination point.

\section{A. Designing a switch unit}

The switch unit continually determines the situation of each agent, and promptly activates and switches agents. To accomplish this task, a data base is composed consisting of the information obtained from the ultra-sound distance sensors 
representing the emerging situation of unknown static environment. Obstacle-bypass agent is activated when one of the six sensors US2-IR2, US3-IR3, US4-IR4 or US7-IR7, US8-IR8, US9-IR9 detects an obstacle. Wall tracking agent is activated when the obstacle i detected by all four sensors US1-IR1, US10-IR10 or US5-IR5, US6-IT6 located on the left or on the right side of the mobile robot. Movement to destination agent is described when there are no obstacles in the operation zone of infrared or ultra-sound sensors around the robot. Switch unit operation modes are presented in the table 1, where:

- 0 - no obstacles in the zone of sensor (both ultra-sound and infra-red) operation detected;

- 1 - any obstacle is detected;

- 2 - irrelevant.

Table 1. Switch unit operation modes

\begin{tabular}{|c|c|c|c|c|c|c|c|c|c|c|c|}
\hline Mode & $d_{S 10}$ & $d_{S I}$ & $d_{S 2}$ & $d_{S 3}$ & $d_{S 4}$ & $d_{S 5}$ & $d_{S 6}$ & $d_{S 7}$ & $d_{S 8}$ & $d_{S \varphi}$ & Agent \\
\hline 1 & 0 & 0 & 0 & 0 & 0 & 0 & 0 & 0 & 0 & 0 & Movement to destination \\
\hline 2 & 0 & 1 & 0 & 0 & 0 & 1 & 0 & 0 & 0 & 0 & Movement to destination \\
\hline 3 & 0 & 1 & 0 & 0 & 0 & 0 & 0 & 0 & 0 & 0 & Movement to destination \\
\hline 4 & 0 & 0 & 0 & 0 & 0 & 1 & 0 & 0 & 0 & 0 & Movement to destination \\
\hline 5 & 1 & 0 & 0 & 0 & 0 & 0 & 1 & 0 & 0 & 0 & Movement to destination \\
\hline 6 & 1 & 0 & 0 & 0 & 0 & 0 & 0 & 0 & 0 & 0 & Movement to destination \\
\hline 7 & 0 & 0 & 0 & 0 & 0 & 0 & 1 & 0 & 0 & 0 & Movement to destination \\
\hline 8 & 1 & 1 & 0 & 0 & 0 & 0 & 0 & 0 & 0 & 0 & Wall tracking \\
\hline 9 & 0 & 0 & 0 & 0 & 0 & 1 & 1 & 0 & 0 & 0 & Wall tracking \\
\hline 10 & 1 & 1 & 0 & 0 & 0 & 1 & 1 & 0 & 0 & 0 & Wall tracking \\
\hline 11 & 1 & 1 & 0 & 0 & 0 & 1 & 2 & 0 & 0 & 0 & Wall tracking \\
\hline 12 & 2 & 1 & 0 & 0 & 0 & 1 & 1 & 0 & 0 & 0 & Wall tracking \\
\hline 13 & 2 & 2 & 0 & 0 & 0 & 1 & 1 & 0 & 0 & 0 & Wall tracking \\
\hline 14 & 1 & 1 & 0 & 0 & 0 & 2 & 2 & 0 & 0 & 0 & Wall tracking \\
\hline 15 & 0 & 0 & 0 & 0 & 0 & 0 & 0 & 1 & 2 & 2 & Obstacle bypass \\
\hline 16 & 0 & 0 & 0 & 0 & 0 & 0 & 0 & 2 & 1 & 2 & Obstacle bypass \\
\hline 17 & 0 & 0 & 0 & 0 & 0 & 0 & 0 & 2 & 2 & 1 & Obstacle bypass \\
\hline 18 & 0 & 0 & 0 & 0 & 0 & 0 & 0 & 1 & 1 & 2 & Obstacle bypass \\
\hline 19 & 0 & 0 & 0 & 0 & 0 & 0 & 0 & 1 & 2 & 1 & Obstacle bypass \\
\hline 20 & 0 & 0 & 0 & 0 & 0 & 0 & 0 & 2 & 1 & 1 & Obstacle bypass \\
\hline 21 & 0 & 0 & 0 & 0 & 0 & 0 & 0 & 1 & 1 & 1 & Obstacle bypass \\
\hline 22 & 2 & 2 & 1 & 2 & 2 & 2 & 2 & 2 & 2 & 2 & Obstacle bypass \\
\hline 23 & 2 & 2 & 2 & 1 & 2 & 2 & 2 & 2 & 2 & 2 & Obstacle bypass \\
\hline 24 & 2 & 2 & 2 & 2 & 1 & 2 & 2 & 2 & 2 & 2 & Obstacle bypass \\
\hline 25 & 2 & 2 & 1 & 1 & 2 & 2 & 2 & 2 & 2 & 2 & Obstacle bypass \\
\hline 26 & 2 & 2 & 1 & 2 & 1 & 2 & 2 & 2 & 2 & 2 & Obstacle bypass \\
\hline 27 & 2 & 2 & 2 & 1 & 1 & 2 & 2 & 2 & 2 & 2 & Obstacle bypass \\
\hline 28 & 2 & 2 & 1 & 1 & 1 & 2 & 2 & 2 & 2 & 2 & Obstacle bypass \\
\hline
\end{tabular}

\section{B. Development of fuzzy block models for mobile robot movement planning system}

The structure of an intelligent system for planning mobile robot movement in an unknown static environment (Figure 1) is composed of four fuzzy blocks. The first three fuzzy blocks 1FB, 2FB, 3FB are used to determine mobile robot rotation angles $\mu_{1}, \mu_{2}$ and $\mu_{3}$ which corresponds to obstacle bypass, wall tracking and movement-to-destination agents. The last fuzzy block $4 \mathrm{FB}$ is intended for controlling the speed of robot motors.

Mobile robot rotation angle $\mu_{1}$ in the obstacle bypass agent is determined with the help of the first fuzzy block 1FB based on the information obtained from any of the sensors US2-IR2, US3-IR3, US4-IR4 or US7-IR7, US8-IR8, US9-IR9. In addition, inputs to $1 \mathrm{FB}$ are the signals $\mathrm{d}_{\mathrm{S} 2}, \mathrm{~d}_{\mathrm{S} 3}$, $\mathrm{d}_{\mathrm{s} 4}$ ir $\mathrm{d}_{\mathrm{S} 7}, \mathrm{~d}_{\mathrm{s} 8}$, $\mathrm{d}_{\mathrm{s} 9}$ of the sensors US2-IR2, US3-IR3, US4-IR4 or US7-IR7, US8IR8, US9-IR9.. Output of 1FB is the mobile robot rotation angle $\mu_{1}$.

Inputs to 1FB are described by four dependency functions (DF): two of them are trapezoidal: $\mathrm{C}$ (close to the robot) and $\mathrm{F}$ (far from the robot); two - triangles: MD (medium distance from the robot) and MF (moderately far from the robot).

Outputs to 1FB are determined by seven dependency functions (DF) with values from -120 to +120 degrees. Two of them are trapezoidal BSR (big step to the right) and BSL (big step to the left), and five triangular: MSR (moderate step to the right), SSR (small step to the right), Z (zero), SSL (small step to the left), MSL (moderate step to the left).

Figure 2 presents the diagrams of input and output dependency functions for the 1FB which is dedicated to the obstaclebypass agent. 1FB fuzzy rule bases are presented in the Table 2 . 

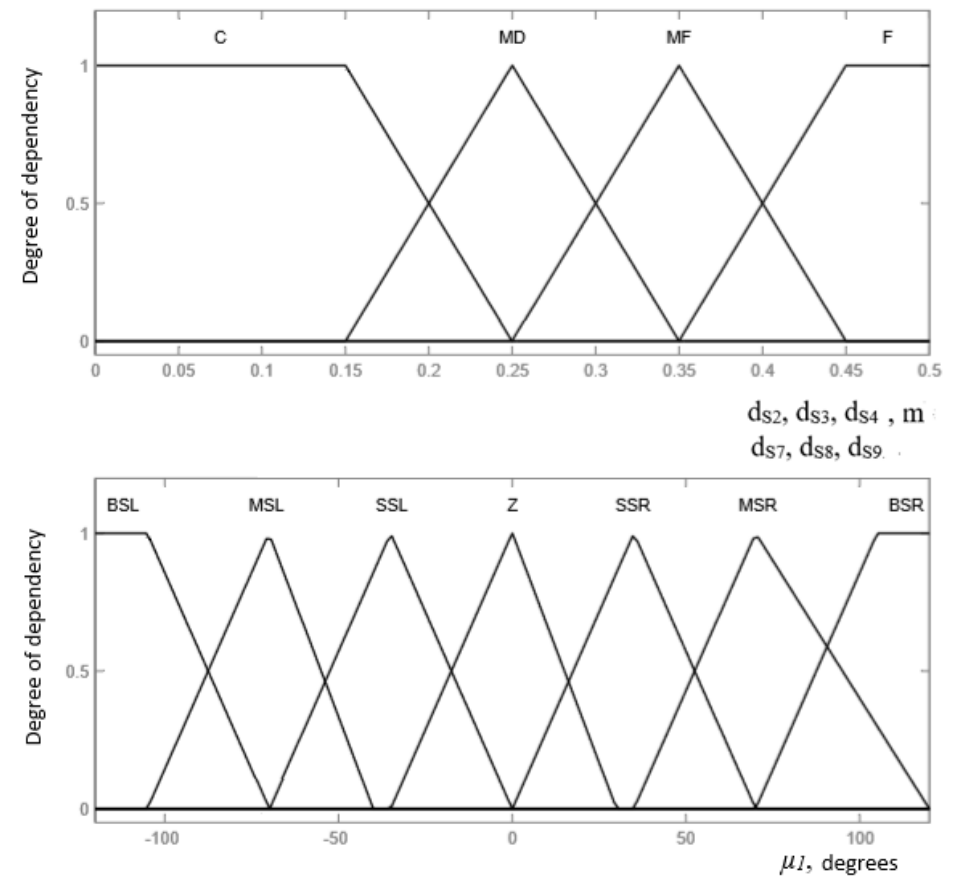

Figure 2. Diagrams of input and output dependency functions for the 1FB dedicated to the obstacle-bypass agent.

If $d_{\$ 4}=\mathrm{C}$

If $d_{s 4}=\mathrm{MD}$

If $d_{s 4}=\mathrm{MF}$

If $d_{S 4}=\mathrm{F}$

Table 2. Fuzzy rule bases for $1 \mathrm{FB}$ dedicated to the obstacle-bypass agent with output parameter $\mu_{1}$ :

\begin{tabular}{|c|c|c|c|c|c|}
\hline \multicolumn{2}{|c|}{$\boldsymbol{\mu}_{\boldsymbol{I}}$} & \multicolumn{4}{c|}{$\boldsymbol{d}_{\boldsymbol{S} 3}$} \\
\cline { 3 - 6 } & $\mathbf{C}$ & BSR & BSR & BSR & BSR \\
\hline \multirow{4}{*}{$\boldsymbol{d}_{\mathbf{S} 2}$} & MD & BSR & BSR & BSR & BSR \\
\cline { 2 - 6 } & MF & BSR & BSR & BSR & BSR \\
\cline { 2 - 6 } & F & BSR & BSR & BSR & BSR \\
\hline
\end{tabular}

\begin{tabular}{|c|c|c|c|c|c|}
\hline \multicolumn{2}{|c|}{$\boldsymbol{\mu}_{\boldsymbol{1}}$} & \multicolumn{4}{c|}{$\boldsymbol{d}_{\mathbf{3}}$} \\
\cline { 3 - 6 } & $\mathbf{C}$ & BSR & BSR & BSR & BSR \\
\hline \multirow{4}{*}{$\boldsymbol{d}_{\boldsymbol{2} 2}$} & MD & BSR & BSR & BSR & BSR \\
\cline { 2 - 6 } & MF & BSR & BSR & MSR & MSR \\
\cline { 2 - 6 } & F & BSR & BSR & MSR & MSR \\
\hline
\end{tabular}

\begin{tabular}{|c|c|c|c|c|c|}
\hline \multicolumn{2}{|c|}{$\boldsymbol{\mu}_{\boldsymbol{I}}$} & \multicolumn{4}{|c|}{$\boldsymbol{d}_{\boldsymbol{S} 3}$} \\
\cline { 3 - 6 } & $\mathbf{C}$ & BSR & BSR & BSR & BSR \\
\hline \multirow{4}{*}{$\boldsymbol{d}_{S 2}$} & MD & BSR & BSR & BSR & BSR \\
\cline { 2 - 6 } & MF & BSR & MSR & MSR & MSR \\
\cline { 2 - 6 } & $\mathbf{F}$ & BSR & MSR & MSR & SSR \\
\hline
\end{tabular}

\begin{tabular}{|c|c|c|c|c|c|}
\hline \multirow{2}{*}{\multicolumn{2}{|c|}{$\boldsymbol{\mu}_{1}$}} & \multicolumn{4}{|c|}{$d_{s 3}$} \\
\hline & & $\mathrm{C}$ & MD & MF & $\mathbf{F}$ \\
\hline \multirow{4}{*}{$d_{S 2}$} & $\mathbf{C}$ & BSR & BSR & BSR & BSR \\
\hline & MD & BSR & BSR & BSR & BSR \\
\hline & MF & BSR & MSR & MSR & MSR \\
\hline & $\mathbf{F}$ & BSR & MSR & SSR & $\mathrm{Z}$ \\
\hline
\end{tabular}

Fuzzy logical inference is made using Mamdani's method, and the outputs of fuzzy blocks are calculated by defuzzification applying the weight centre method.

To determine mobile robot rotation angle $\mu_{2}$ the second fuzzy block $2 \mathrm{FB}$ is used in the wall tracking agent. As it was already mentioned, the sensors of this agent US2-IR2, US3-IR3, US4-IR4 or US7-IR7, US8-IR8, US9-IR9 do not detect obstacles, and the sensors located on the same side (US1-IR1, US10-IR10 or US5-IR5, US6-IR6), do detect obstacles (or a 
group of obstacles). Moreover, mobile robot moves along the edge of an obstacle from the left or from the right. Inputs to $2 \mathrm{FB}$ are the signals of the sensors US1-IR1, US10-IR10 or US5-IR5, US6-IR6: $d_{S I}$ and $d_{S I 0}$ or $d_{S 5}$ and $d_{S 6}$ NB-2 output: mobile robot rotation angle $\mu_{2}$.

Inputs to $1 \mathrm{FB}$ are described by four dependency functions (DF): two of them are trapezoidal: $\mathrm{C}$ (close to the robot) and F (far from the robot); two - triangles: MD (medium distance from the robot) and MF (moderately far from the robot).

Output $\mu_{2}$ 2FB is described by seven dependency functions (DF). Two of them are trapezoidal: BSR (big step to the right) and BSL (big step to the left), and five triangular: MSR (moderate step to the right), SSR (small step to the right); Z (zero), SSL (small step to the left), MSL (moderate step to the left).

Figure 3. Presents the diagrams of input and output dependency functions for the $2 \mathrm{FB}$ which is dedicated to the wall tracking agent.
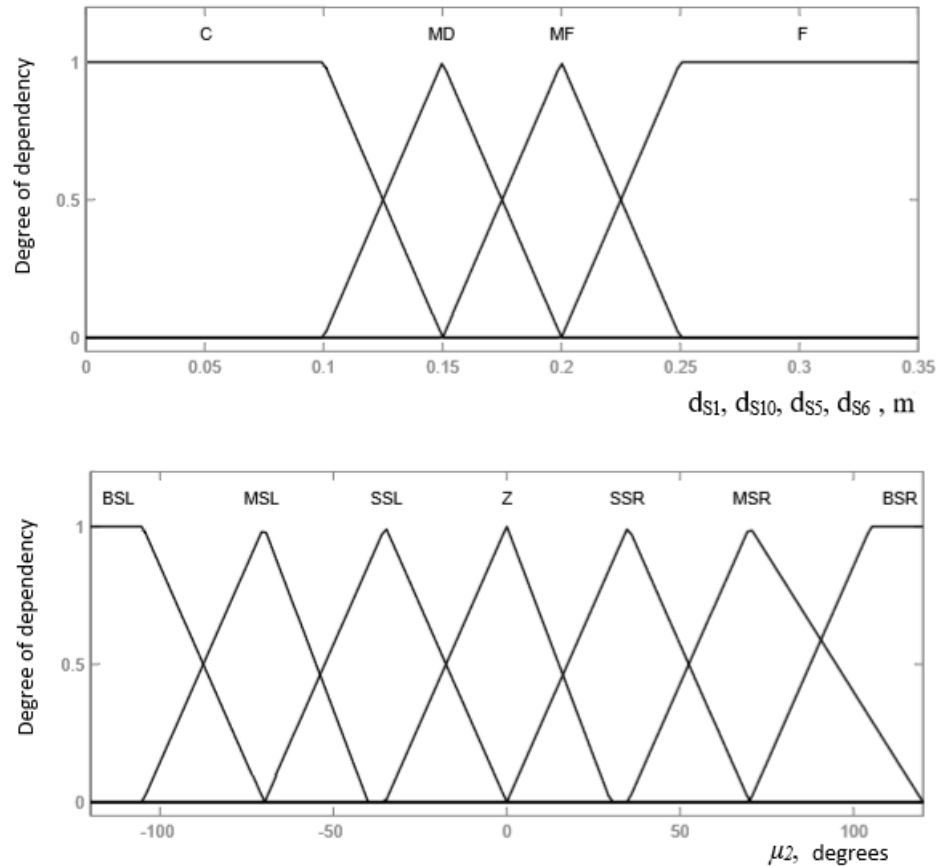

Figure 3. Diagrams of input and output dependency functions for the 2FB which is dedicated to the wall tracking agent.

2FB fuzzy rule bases are presented in the Table 3.

Table 3. Fuzzy rule bases for $2 \mathrm{FB}$ dedicated to the obstacle-bypass agent with output parameter $\mu_{2}$ :

When tracked from the right

\begin{tabular}{|c|c|c|c|c|c|}
\hline \multirow{2}{*}{\multicolumn{2}{|c|}{$\boldsymbol{\mu}_{2}$}} & \multicolumn{4}{c|}{$\boldsymbol{d}_{55}$} \\
\cline { 3 - 6 } & $\mathbf{C}$ & SSL & MD & MF & F \\
\hline \multirow{4}{*}{$\boldsymbol{d}_{\mathbf{S} 6}$} & MD & SSL & Z & MSR & MSR \\
\cline { 2 - 6 } & MF & MSL & SSL & SSR & MSR \\
\cline { 2 - 6 } & F & MSL & MSL & Z & MSR \\
\cline { 2 - 6 } & \multicolumn{4}{|c}{} \\
\hline
\end{tabular}

When tracked from the left

\begin{tabular}{|c|c|c|c|c|c|}
\hline \multicolumn{2}{|c|}{$\boldsymbol{\mu}_{2}$} & \multicolumn{4}{c|}{$\boldsymbol{d}_{S 1}$} \\
\cline { 3 - 6 } & $\mathbf{C}$ & SSR & SSL & MSL & MSL \\
\hline \multirow{4}{*}{$\boldsymbol{d}_{\text {SI0 }}$} & MD & SSR & Z & SSL & MSL \\
\cline { 2 - 6 } & MF & MSR & SSR & SSL & MSL \\
\cline { 2 - 6 } & F & MSR & MSR & Z & MSL \\
\hline
\end{tabular}

The third fuzzy block 3FB is used to determined mobile robot rotation angle in the movement-to-destination agent. As it was already mentioned, movement-to-destination agent is activated when the sensors US2-IR2, US3-IR3, US4-IR4 or US7IR7, US8-IR8, US9-IR9 do not detect any obstacle, and sensors located on the same side (US1-IR1, US10-IR10 or US5-IR5, US6-IR6) do not detect obstacle either. In addition, input to 3FB is $\theta$ - the angle between the mobile robot rotation direction $(\phi)$ and the direction of the destination point direction $(\beta)$ (Fig. 4). Mobile robot rotation angle $\mu_{3}-3$ FB output. 
Output to 3FB $\theta$ is described by seven dependency functions (DF), measured in degrees (from -150 to $+150^{\circ}$ ): two of them being trapezoidal: FR (far on the right from the destination) and FL (far on the left from the destination); two of them being triangular: MFR (moderately far on the right from the destination), CR (Close on the right to the destination), Z (zero), $\mathrm{CL}$ (close on the left to the destination), MFL (moderately far on the left from the destination).

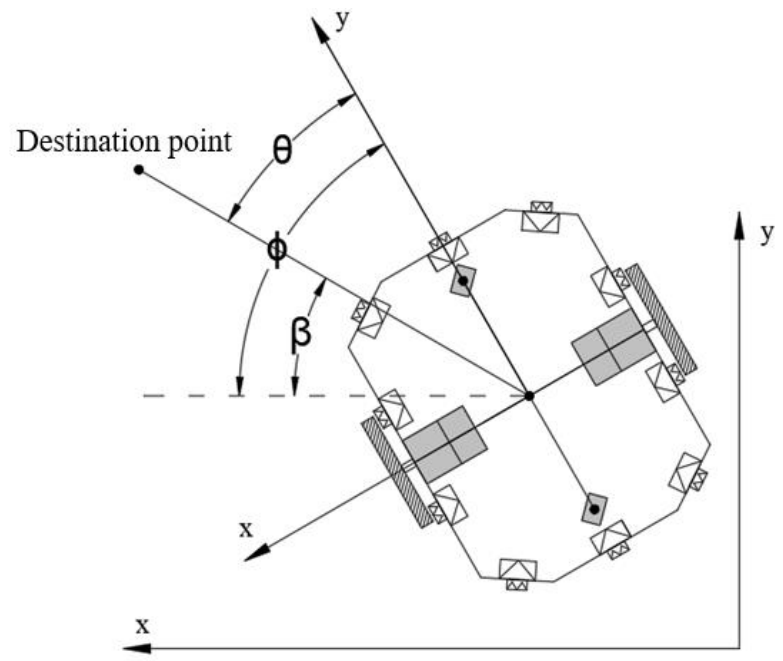

Figure 4. Scheme of robot navigation to determine the NB-3 input parameter.

If $(\varphi-\beta)>180^{\circ}$ then $\theta=\varphi-\beta-360^{\circ}$.

If $(\varphi-\beta)<180^{\circ}$ then $\theta=\varphi-\beta+360^{\circ}$.

In addition, $\theta=\varphi-\beta^{\circ}$, where $0^{\circ}<\varphi, \beta<360^{\circ}$ and $-180^{\circ}<0<+180^{\circ}$ (Sandeep, 2016; Mousavi, 2015).

3FB output $\mu_{3}$ is described by seven dependency functions (DF) with values from -120 to +120 degrees. Two of them are trapezoidal: BSR (big step to the right) and BSL (big step to the left), and five triangular: MSR (moderate step to the right), SSR (small step to the right); Z (zero), SSL (small step to the left), MSL (moderate step to the left).

Fig 5 presents the diagrams of input and output dependency functions for the 3FB which is dedicated to the movementto-destination agent.

3FB fuzzy rule bases are presented in the Table 4.
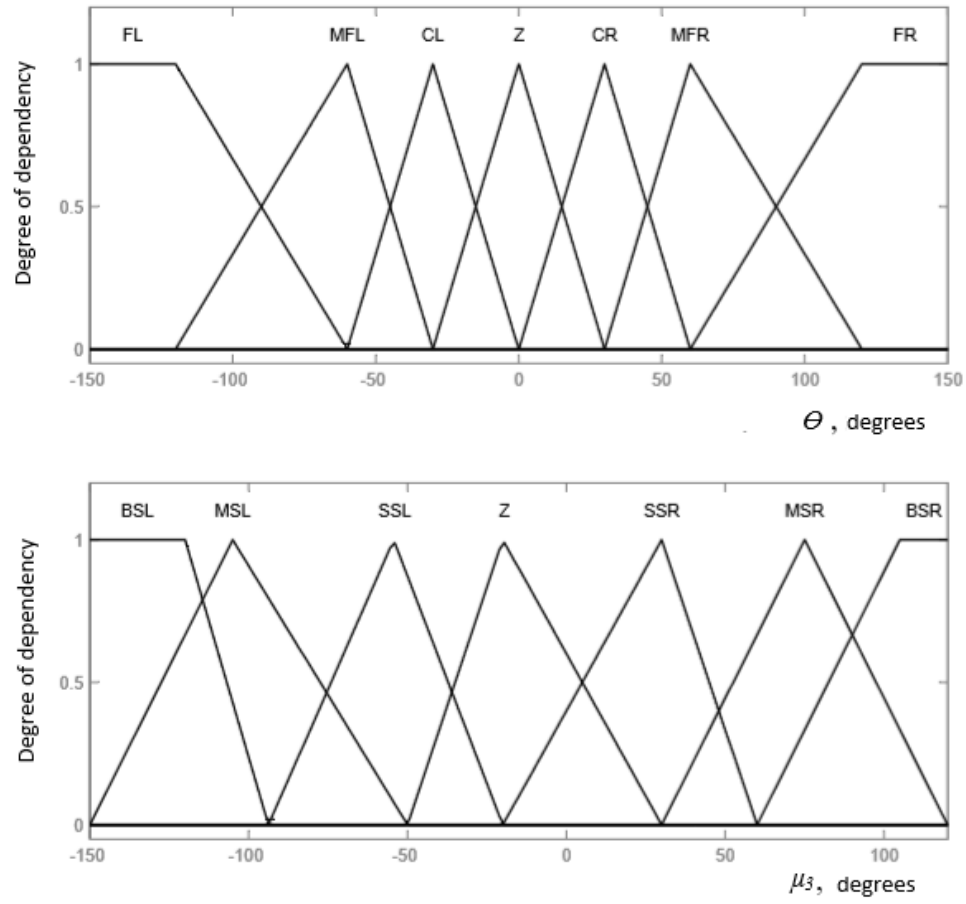

Figure 5. Diagrams of input and output dependency functions for the 3FB which is dedicated to the movement-to-destination agent. 
Table 4. Fuzzy rule bases for 3FB dedicated to the movement to destination agent with the input parameter $\mu_{3}$.

\begin{tabular}{|l|c|c|c|c|c|c|c|}
\hline $\boldsymbol{\theta}$ & FL & MFL & CL & Z & CR & MFR & FR \\
\hline $\boldsymbol{\mu}_{3}$ & BSL & MSL & SSL & Z & SSR & MSR & BSR \\
\hline
\end{tabular}

Motor speed control plays an important role in planning mobile robot movement in an unknown dynamic environment. In order for a mobile robot to move without colliding with obstacles and reach the destination, the ratio between the mobile robot rotation angle $\mu\left(\mu_{1}, \mu_{2}\right.$ and $\mu_{3}$ outputs of the fuzzy blocks 1FB, 2FB and 3FB are involved), the distance between the robot and the destination point and the average velocity of the mobile robot $V_{a v g}$ are presented using the fourth fuzzy block $4 \mathrm{FB}$.

Two 4FB inputs are the mobile robot rotation angle $\mu$ and the distance from the robot to the direction point. 4FB output: average mobile robot speed $V_{a v g}$.

Mobile robot rotation angle $\mu$ is described by seven dependency functions (DF), measured in degrees (from -120 to $+120^{\circ}$ ). Two of them are trapezoidal: BSR (big step to the right) and BSL (big step to the left), and five triangular: MSR (moderate step to the right), SSR (small step to the right); Z (zero), SSL (small step to the left), MSL (moderate step to the left).

The distance from the robot to the destination point $d_{\text {destination }}$ is described by four dependency functions (DF), measured in metres (from 0 to 0.5 ): one of the being trapezoidal: $\mathrm{F}$ (far from the robot); and three triangular: $\mathrm{C}$ (close to the robot), MD (at the moderate distance from the robot) and MF (moderately far from the robot).

4FB output, $V_{a v g}$ is determined by four triangular dependency functions (DF) with values varying from 0 to $V_{\text {max }}: \mathrm{Z}$ (zero), S (slow), M (moderate) and $\mathrm{H}$ (high).
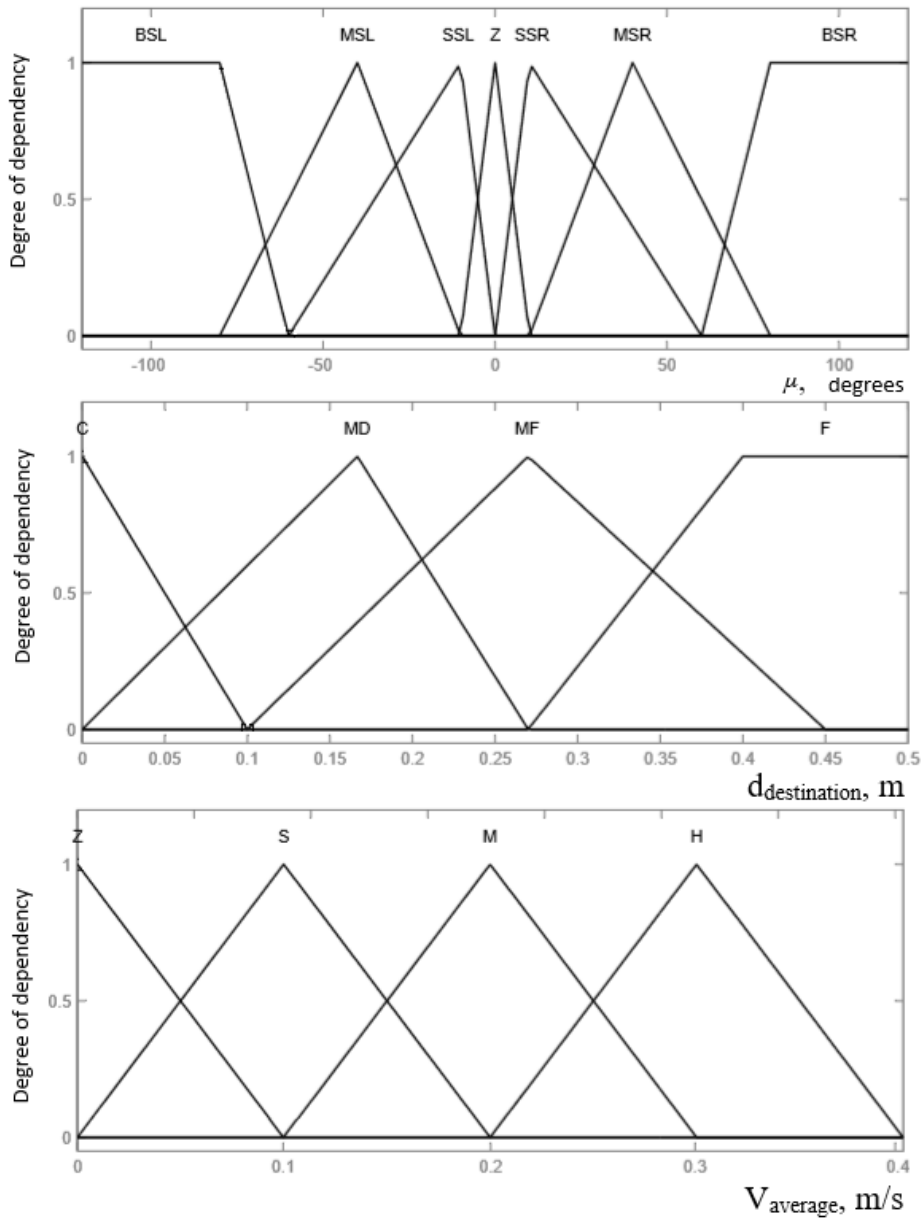

Figure 6. Diagrams of input and output dependency functions for the 4FB which is dedicated to the speed control agent.

Figure 6 presents the diagrams of input and output dependency functions for the 4FB which is dedicated to the speed control agent.

4FB fuzzy rule bases are presented in the Table 5.

Table 5. Fuzzy rule bases for 4FB dedicated to speed control agent with the input parameter $V_{a v g}$. 
Table 5. Fuzzy rule bases for 4FB dedicated to speed control agent with the input parameter $\mathrm{V}_{\text {avg }}$

\begin{tabular}{|c|c|c|c|c|c|}
\hline \multirow{2}{*}{\multicolumn{2}{|c|}{$V_{\text {avg }}$}} & \multicolumn{4}{c|}{$\boldsymbol{d}_{\text {destination }}$} \\
\cline { 3 - 6 } & C & MD & MF & F \\
\hline \multirow{4}{*}{$\boldsymbol{\mu}$} & BSL & Z & Z & S & M \\
\cline { 2 - 6 } & MSL & Z & Z & S & M \\
\cline { 2 - 6 } & SSL & Z & S & M & H \\
\cline { 2 - 6 } & Z & Z & S & M & H \\
\cline { 2 - 6 } & SSR & Z & S & M & H \\
\cline { 2 - 6 } & MSR & Z & Z & S & M \\
\cline { 2 - 6 } & BSR & Z & Z & S & M \\
\hline
\end{tabular}

\section{RESULTS AND DISCUSSION}

To verify the accuracy of the intelligent system intended for planning robot movement in an unknown environment using a multi-agent system, computer simulation of the system is performed based on the theoretical analysis and research results. For computer simulation performed in the V-REP environment.

Simulation of mobile robot movement involves three modes which correspond to three agents (obstacle-bypass, wall tracking, and movement-to-destination). The values of the developed fuzzy blocks (1FB - for obstacle-bypass; $2 \mathrm{FB}$ - for wall tracking agent; $3 \mathrm{FB}$ - for movement-to-destination agent, and 4FB - for motor speed control agent).

A. Simulation of mobile robot movement planning intelligent system in the obstacle-bypass agent

In the obstacle-bypass agent, mobile robot movement is planned using information from any of the sensors US2-IR2, US3-IR3, US4-IR4 or US7-IR7, US8-IR8, US9-IR9 and two fuzzy blocks 1FB and 4FB. For the 1FB developed to determine the mobile robot rotation angle in the obstacle-bypass agent, the values of the distance between the sensors and the mobile robot rotation angle $\mu_{l}$ correspond to the diagrams presented in the Fig. 2 Values of $4 \mathrm{NB}$, which is dedicated to motor speed control, are presented in the Fig. 6 . The diagram values of the fuzzy blocks' dependency function are presented in tables 2 and 6 .

When testing the obstacle-bypass agent, mobile robot moves from the starting point $\mathrm{A}\left(\mathrm{x}_{1}=0.500, \mathrm{y}_{1}=0.500\right)$ to the destination point $\mathrm{B}\left(\mathrm{x}_{2}=8.000, \mathrm{y}_{2}=3.000\right)$, and there is an obstacle on its way (Fig. 7). After 769 iterations of the program (the set time is $19.60 \mathrm{~s}$ ) the mobile robot reached the destination without colliding with the obstacle.

In this case, if the sensors do not detect an obstacle, the robot moves forward towards the destination. If any of the sensors US2-IR2, US3-IR3, US4-IR4 or US7-IR7, US8-IR8, US9-IR9 detects obstacles, when obstacles are circumvented using fuzzy blocks $1 \mathrm{FB}$ and $4 \mathrm{NB}$, mobile robot rotation angle $\mu_{l}$ varies. Variation of the rotation angle $\mu_{l}$ depends on the distance between the robot and the destination as well as the relative position. The distance between the robot and the destination reduces to zero; the robot reaches the destination without colliding eith the obstacles. The diagrams of testing parameters including $\mu_{1}, V_{\text {average, }} d_{\text {destination, }}$ are presented in Fig. 7.
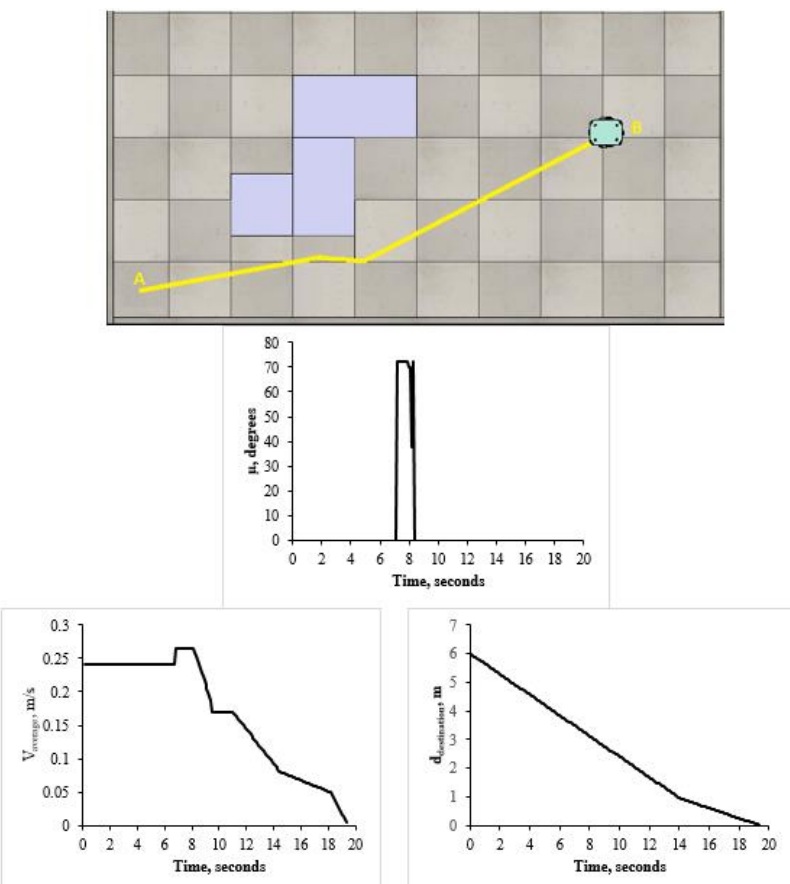

Fig. 7. Results of testing the mobile robot movement intelligent system in the obstacle-bypass agent. 


\section{B. Simulation of mobile robot movement planning intelligent system in the wall tracking agent}

Wall tracking sensors US2-IR2, US3-IR3, US4-IR4 or US7-IR7, US8-IR8, US9-IR9 do not detect an obstacle, and sensors located on the same side (US1-IR1, US10-IR10 or US5-IR5, US6-IR6) detects an obstacle - a wall or obstacles located close to one another. Fuzzy blocks $2 \mathrm{FB}$ and 4FB are used to determine mobile robot rotation angle $\mu_{2}$ in the wall tracking agent and the motor speed control agent. The values of the linguistic variables of the fuzzy blocks $2 \mathrm{FB}$ and 4FB and their dependency functions correspond to the diagrams presented in figures 3 and 6 . The diagram values of the fuzzy blocks' dependency function are presented in tables 3 and 6.

Figure 8 presents the results of the first testing, when the robot moves from the starting point $\mathrm{A}\left(\mathrm{x}_{1}=1.900 ; \mathrm{y}_{1}=0.400\right)$ along a curvy wall. Sensors US1-IR1 and US10-IR10 detect obstacles, and the robot maintains a safe distance to the wall. It must be noted that in order to analyse the movement of a mobile robot, the distance between the mobile robot and the destination point $\mathrm{d}_{\text {destination }}$ was constant during the first testing, and in this case, reaching the destination was not the major goal.

Variation of the values of the mobile robot rotation angle $\mu_{2}$, its average speed $\mathrm{V}_{\text {average }}$, distance from the sensors US1IR1 and US10-IR10 to the wall $\mathrm{d}_{\mathrm{S} 1}, \mathrm{~d}_{\mathrm{S} 10}$ presented in the diagrams in Fig. 8. Changes in the shape of the wall result in constantly varying angle $\mu_{2}$; average velocity $\mathrm{V}_{\text {average }}$ - constant with minimum variation; during the testing, the robot maintains a safe distance to the wall $\left(\mathrm{d}_{\mathrm{S} 1}, \mathrm{~d}_{\mathrm{S} 10}\right.$ from 0.30 to $\left.0.35 \mathrm{~cm}\right)$. After the sensors US1-IR1 and US10-IR10 detect a wall, the final result does not change. Mobile robot moves along the wall maintaining a safe distance.

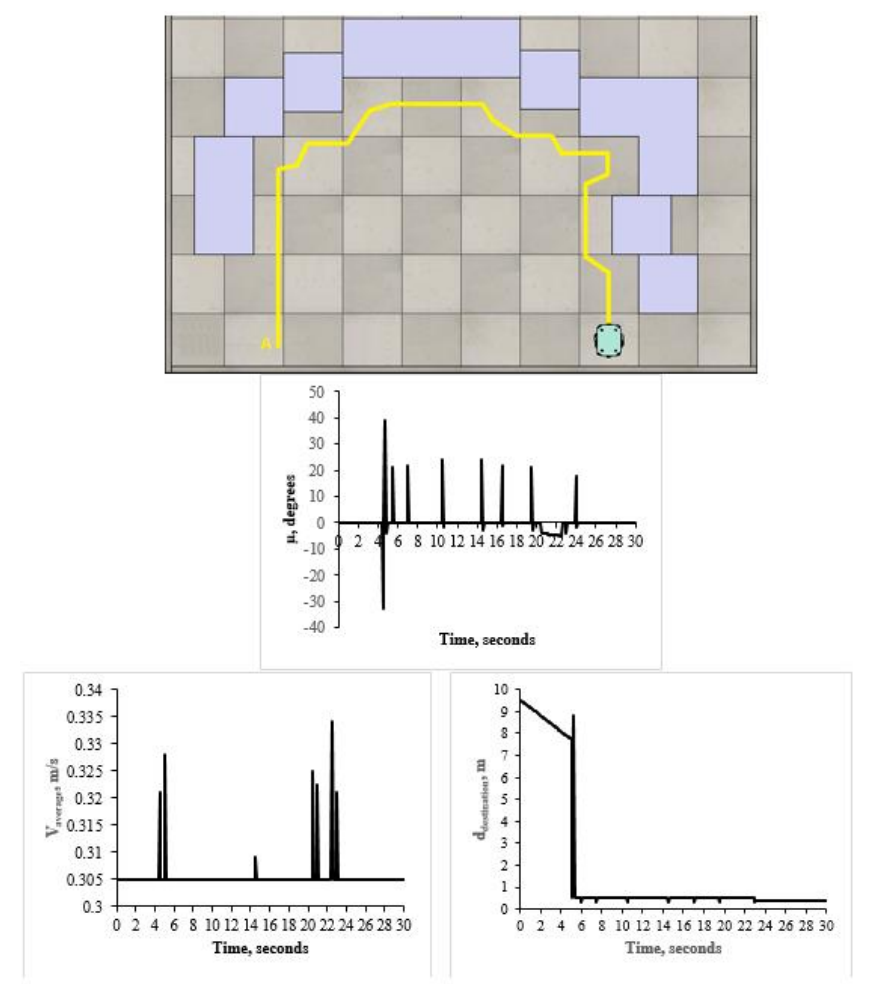

Figure 8. Results of the testing of the mobile robot movement planning intelligent system in the wall tracking agent.

\section{Simulation of mobile robot movement planning intelligent system in the movement-to-destination agent}

In the movement-to-destination agent, there are no obstacles around the robot; fuzzy blocks 3FB and 4FB are used. The values of the angle $\theta$ between the mobile robot direction and destination point direction and the mobile robot rotation angle $\mu_{3}$ for $3 \mathrm{FB}$, which was developed to determine the mobile robot rotation angle in the movement-to-destination agent, correspond to the ones presented in the Fig 5. Values of 4NB, which is dedicated to motor speed control, are presented in the Fig 6. The diagram values obtained by fuzzy blocks' dependency functions are presented in tables 5 and 6.

During the first testing, mobile robot was moving from the starting point $\mathrm{A}\left(\mathrm{x}_{1}=1.000 ; \mathrm{y}_{1}=1.000\right)$ to the destination point $\left(\mathrm{x}_{2}=1.000 ; \mathrm{y}_{2}=4.00\right)(\mathrm{Fig} 9)$. After 428 iterations of the program (the set time is $\left.11.9 \mathrm{~s}\right)$ the mobile robot reached the destination.

During the first testing, destination point is located on the robot's movement trajectory, therefore, during simulation, the mobile robot rotation angle $\mu_{3}$ did not change, and the robot was moving forward to the destination. Average velocity $\mathrm{V}_{\text {average }}$ was dropping, and the distance between the mobile robot and the destination point $\mathrm{d}_{\text {destination }}$ eventually became equal to zero, i.e. the robot reached the destination point. 


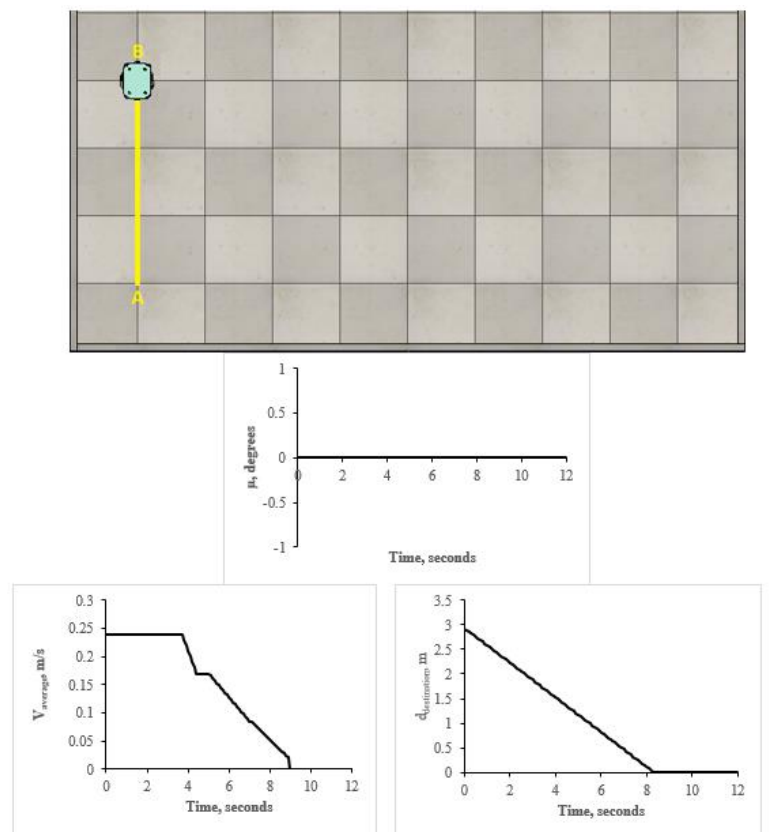

Figure 9. Results of the first testing of the mobile robot movement planning intelligent system in the movement-to-destination agent.

D. Simulation of an intelligent system for planning mobile robot movement in an unknown static environment

While performing the testing, mobile robot was moving from the starting point $\mathrm{A}\left(\mathrm{x}_{1}=3,000 ; \mathrm{y}_{1}=0,500\right)$ to the destination point $\mathrm{B}\left(\mathrm{x}_{2}=6.000 ; \mathrm{y}_{2}=4.000\right)$. Several obstacles were located in the operating area. After 1142 iterations of the program (over $29.34 \mathrm{~s}$ ) the mobile robot reached the destination. The movement trajectory - result of the testing - is presented in the Fig. 10 and include five sub-trajectories. Sub-trajectories are separated from one another by switching points (P1, P2, P3, P4):

- from the starting point A to P1: sub-trajectory of the movement-to-destination agent;

- from P1 to P2: sub-trajectory of the wall tracking agent;

- from P2 to P3: sub-trajectory of the movement-to-destination agent;

- from P3 to P4: sub-trajectory of the wall tracking agent;

- from P4 to the destination point B: sub-trajectory of the movement-to-destination agent;
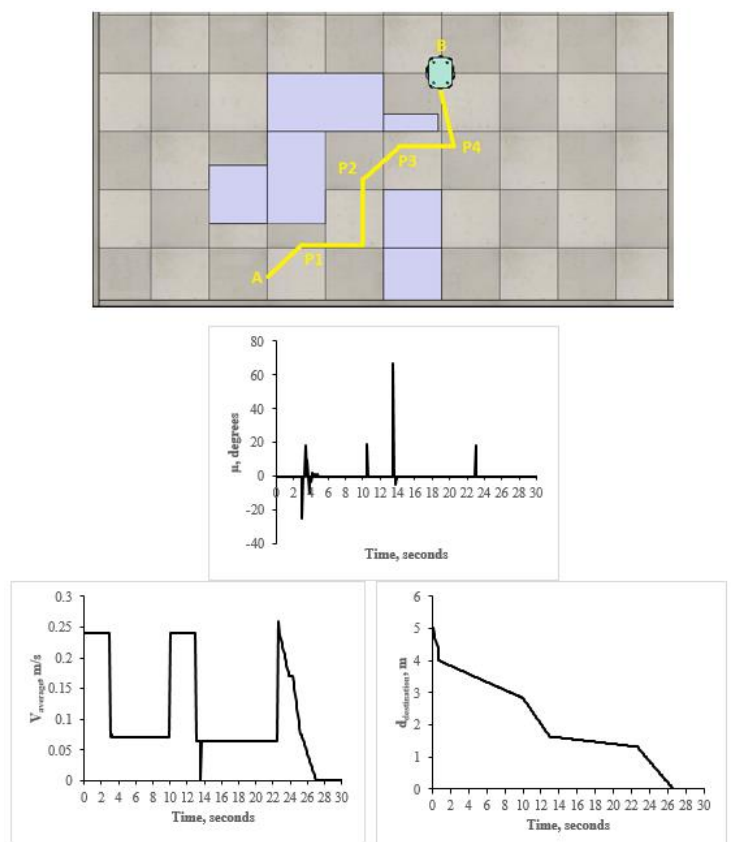

Figure 10. Results of testing the intelligent system for planning mobile robot movement in an unknown environment

At the point P1, the intelligent system switches the movement-to-destination agent to the obstacle-bypass agent, and later to the wall-tracking agent. Similarly, the wall-tracking agent is switched to the movement-to-destination agent 
at the point P2; At the point P3, the movement-to-destination agent is switched to the obstacle-bypass agent, and then to the wall-tracking agent. At the point P4, the wall-tracking agent is switched to the movement-to-destination agent.

Variations of the values of the mobile robot rotation angle $\mu$, mobile robot average velocity $\mathrm{V}_{\text {average }}$ and the distance between the robot and the destination point are presented in the diagrams in the Fig. 10. It can be seen that the values of $\mu$ and $\mathrm{V}_{\text {average }}$ significantly changes at switching point.

\section{CONCLUSIONS}

After accomplishing the analysis and simulation described in the present article, the following conclusions can be made.

While moving in an unknown environment mobile robot performs three tasks: circumvent obstacles, tracks wall, and moves towards the destination point. Therefore, a fuzzy structure comprising of four fuzzy blocks for each of the specified agents, including the safe movement agent, was offered for the development of an intelligent system for planning movement of a mobile robot in an unknown static environment using a multi-agent system. In addition, the first three blocks perform calculation of the preliminary value of the robot rotation angle in the direction of the surrounding obstacles in each of the agents, and the fourth block deals with the problem of how to avoid collision of the robot and the unknown static obstacles located in its operating area. The preliminary rotation angle and the distance between the robot and the destination point are considered as the introductory parameters of the fourth fuzzy block. The output of the second fuzzy block is the final value of the mobile robot average velocity variation.

Application of a multi-agent method for the development of an intelligent planning system is optimal for dealing with the mobile robot movement in an unknown static environment planning task. Dividing complex environment into several simple specific situations corresponding each of the agents reduces the number of input variables and the amount of calculations.

There are different agents, and their choice affects the accuracy and efficiency of the movement planning. Using more agents improves the accuracy, however, it becomes more complicated task to create data bases for fuzzy blocks and dependency functions for each of the agents.

An intelligent system is composed of four main agents (obstacle-bypass, wall-tracking, movement-to-destination, and speed control), which ensures the planning task of a mobile robot movement in a static environment will be accomplished.

\section{REFERENCES}

1. Adib A., Masoumi B. 2017. Mobile robots navigation in unknown environments by using fuzzy logic and learning automata. Proceedings of the International Scientific Conference "2017 Artificial Intelligence and Robotics (IRANOPEN)", Qazvin, Iran, pp. 58-63. https://doi.org/10.1109/RIOS.2017.7956444

2. Ahmad H., Othman N.A. 2016. Investigation on intermittent observation in mobile robot localization with fuzzy logic technique. 2016 IEEE International Conference "Automatic Control and Intelligent Systems (I2CACIS)", Selangor, Malaysia, pp. 17-22. https://doi.org/10.1109/I2CACIS.2016.7885282

3. Berisha J., BajramiX., Shala A., Likaj R. 2016. Application of fuzzy logic controller for obstacle detection and avoidance on real autonomous mobile rob t. $5^{\text {th }}$ Mediterranean Conference on Embedded Computing (MECO), Bar, Montenegro, pp. $200-205$. https://doi.org/10.1109/MECO.2016.7525740

4. Boujelben M., Ayedi D., Rekik C., Derbel N. 2017. Fuzzy logic controller for mobile robot navigation to avoid dynamic and static obstacles. 14 ${ }^{\text {th }}$ International Multi-Conference "Systems, Signals \& Devices (SSD)", Marrakech, Morocco, pp. $293-298$. https://doi.org/10.1109/SSD.2017.8166963

5. Handayani A.S., Dewi T., Husni N.L., Nurmaini S., Yani, I. 2017. Target tracking in mobile robot under uncertain environment using fuzzy logic controller. $4^{\text {th }}$ International Conference "Electrical Engineering, Computer Science and Informatics (EECSI)", Yogyakarta, Indonesia, pp. 1-5. https://doi.org/10.1109/EECSI.2017.8239079

6. Luo C. 2017. Neural-network-based fuzzy logic tracking control of mobile robots. $13^{\text {th }}$ IEEE Conference "Automation Science and Engineering (CASE)", Xi'an, China, pp. 1318-1319. https://doi.org/10.1109/COASE.2017.8256283

7. Mousavi M.A. 2015. A fast fuzzy path tracking controller for mobile robots. $3^{\text {rd }}$ RSI International Conference "Robotics and Mechatronics (ICROM)", Tehran, Iran, pp. 675-680. https://doi.org/10.1109/ICRoM.2015.7367864

8. Nasrinahar A., Chuah J.H. 2016. Effective route planning of a mobile robot for static and dynamic obstacles with Fuzzy Logic. $6^{\text {th }}$ IEEE International Conference "Control System, Computing and Engineering (ICCSCE)", Batu Ferringhi, Malaysia, pp. 34-38. https://doi.org/10.1109/ICCSCE.2016.7893541

9. Sandeep B.S., Supriya P. 2016. Analysis of fuzzy rules for robot path planning. 2016 International Conference "Advances in Computing, Communications and Informatics (ICACCI)", Jaipur, India, pp. 309-314. https://doi.org/10.1109/ICACCI.2016.7732065

10. Thinh N.T., Tuan N.T., Hung L. P. 2016. Predictive controller for mobile robot based on Fuzzy Logic. $3^{\text {rd }}$ International Conference "Green Technology and Sustainable Development (GTSD)", Kaohsiung, Taiwan, pp. 141-144. https://doi.org/10.1109/GTSD.2016.41 